CYBERNETICS AND INFORMATION TECHNOLOGIES • Volume 16, No 6 Special issue with selection of extended papers from 6th International Conference on Logistic, Informatics and Service Science LISS'2016

Sofia 2016

\title{
Improved Chirp Scaling Algorithm for Processing Squinted Mode Synthetic Aperture Sonar Data
}

\author{
Naiqiang Fan ${ }^{1}$, Yingmin Wang ${ }^{2}$, Yarong $L v^{3}$ \\ ${ }^{1}$ Engineering Practice Training Center, Northwestern Ploytechnical University, Xi'an, China \\ ${ }^{2}$ School of Marine Science And Technology, Northwestern Ploytechnical University, Xi 'an, China \\ ${ }^{3}$ Northwestern Ploytechnical University Ming De College, Xi'an, China \\ Emails:fannaiqiang@nwpu.edu.cn ywang@nwpu.edu.cn lvyr@npumd.edu.cn
}

\begin{abstract}
The conventional Chirp Scaling Algorithm is mainly used in side looking or small squint angle mode of synthetic aperture imaging. In application of synthetic sonar, the large squint angle imaging is often required and the range-azimuth coupling is serious. On the basis of studying the principle of the conventional Chirp Scaling Algorithm, we improved the imaging algorithm in large squint mode. We analysed the structure of the echo frequency spectrum and the compensation of phase factor. The improved imaging algorithm designs a more precision of phase compensation factor, and eliminates the high order degree of range and azimuth coupling in the specific mapping band. Any target point is simulated in imaging region by using the improved algorithm. The simulation conclusion showed that the improved Chirp Scaling Algorithm is able to meet the imaging focus and is more suitable to slant imaging as compared with the traditional algorithm.
\end{abstract}

Keywords: Synthetic aperture sonar, Chirp Scaling Algorithm, range-azimuth coupling, secondary range compression, Taylor series, Fresnel assumption.

\section{Introduction}

Synthetic Aperture Sonar (SAS) forms the virtual large aperture based on the movement of the small-aperture sonar array, and obtains high-resolution images of the target areas through imaging algorithms [1]. When the angle between the azimuth beam direction and the sonar platform moving trajectory is exactly $90^{\circ}$ during the imaging process of the synthetic aperture, the imaging is called side-looking imaging. 
Otherwise, when the angle differs from $90^{\circ}$, it is called squint mode imaging. The squint angle presents the angle between the beam pointing direction and the perpendicular line of the moving trajectory of the sonar platform. In the squint mode, adjusting of the beam direction provides the observation of the forward target scene in advance, and the reimaging of the backward target scene, so it has great practical significance.

The SAS imaging algorithms can be generally divided into two categories, the time domain algorithms and the frequency domain algorithms. Among the frequency domain algorithms, the Range-Doppler (RD) algorithm, the Chirp Scaling (CS) Algorithm, and the $\omega K$ Algorithm are the most often used ones. The core idea of these imaging algorithms is to convert $2 \mathrm{D}$ signals into two $1 \mathrm{D}$ signals and process them separately via range-azimuth decoupling. CS Algorithm proposed by $\mathrm{N} \mathrm{a} \mathrm{m} \mathrm{le} \mathrm{r}$ and Runge [2] in 1992 made the high-band SAR imaging theory more mature. Compared to other imaging algorithms according to Chirp_Scaling principle, CS Algorithm achieves better space-variant compensation for the Range Cell Migration (RCM) of the imaging area, and realizes the consistent Range Cell Migration Correction (RCMC) by a phase function. The advantage of CS Algorithm does not refer only to the improved imaging efficiency, but also to the greatly improved image quality in squint mode, which is certainly more valuable.

As the squint angle increases, the range-azimuth coupling becomes more important and the decoupling becomes more difficult. Peng Sui-Yang and others [3-5], firstly removed the time offset in the time domain, and then corrected the RCM consistence at different range by the chirp scaling, without considering the high order curves effects on the azimuth processing. The azimuth nonlinear CS Algorithm proposed by Zhang Shuang-Xi and others [6-8] pushed the limits for the horizontal focus depth, and formed high-resolution images at larger azimuth scenes. However, due to the computation complexity, the two mentioned methods do not meet the realtime SAS requirements. Moreover, Zhang Xuebo and others [9-11] proposed the improved CS Algorithm based on the CS implementation in the multiple sub-array technique in order to solve the non-uniform sampling problem. However, this method ignores the higher order terms in the azimuth processing which has impact on the resolution.

In this paper the improved CS imaging algorithm for highly squint SAS is presented. The improved algorithm provides accurate target focusing and improves the image resolution by compensating the phase error of the band reference point. In addition, the update of the CS compensation factor enables larger band imaging. The proposed method is verified by simulation, and results have shown that better image quality is achieved in high squint mode.

\section{Basic signal coupling model}

In the SAS imaging squint angle is one of the most important parameters. When the squint angle is equal to $0^{\circ}$, the imaging is called side-looking mode imaging. When the squint angle is larger than $0^{\circ}$, a coupling between range and azimuth occurs and the squint angle impact on the imaging should be considered [12]. 


\subsection{Geometrical model of the squint SAS echo}

The geometrical model of the squint SAS imaging is presented in Fig. 1, where the $\theta_{s q}$ is the squint angle, $h$ is the height of the tow fish from the sea bottom, $V$ is the drag speed of the tow fish, $R_{B}$ is the vertical distance between the target and the fish path, $R_{0}$ is the instantaneous inclination range, $t_{m}$ is the azimuth time, and $t_{c}$ is the time deviation between the beam center and the target point.

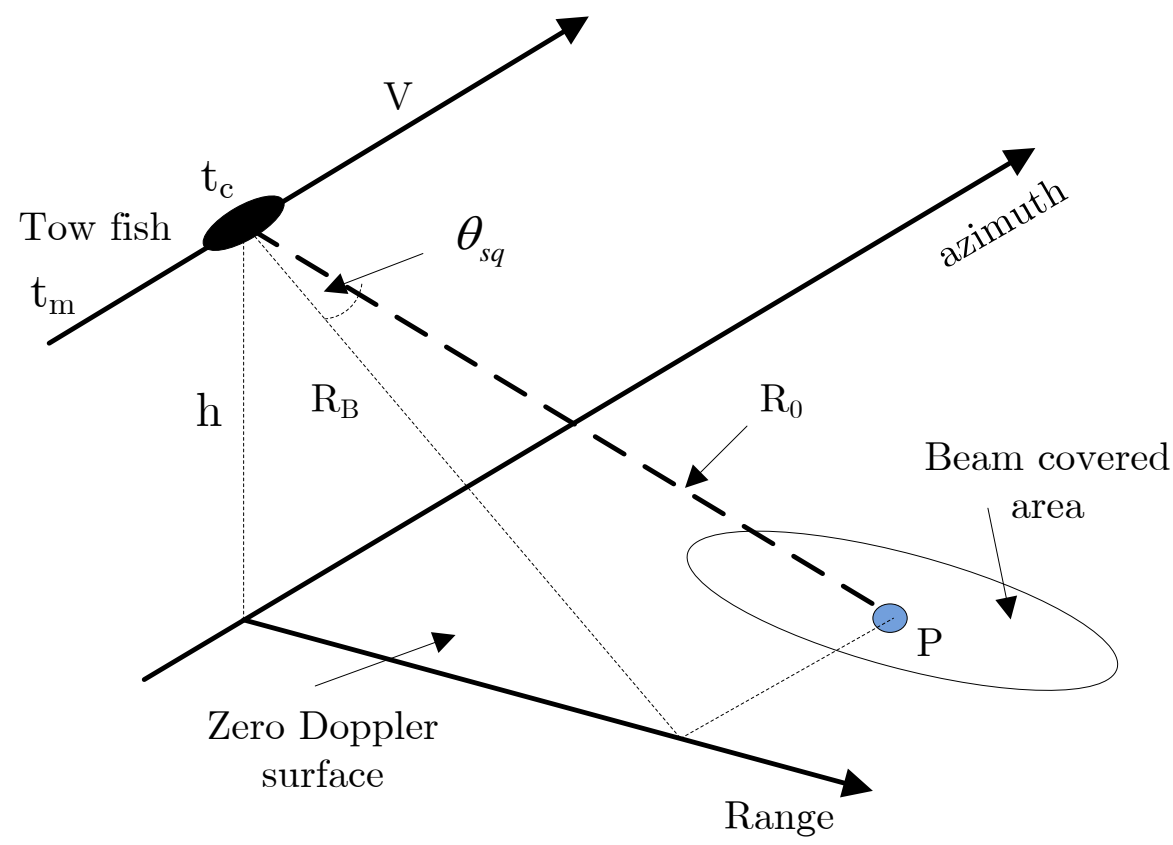

Fig. 1. Geometrical model of the squint SAS

According to the geometrical relations shown in Fig. 1, the instantaneous inclination range is obtained as

$$
\begin{gathered}
R\left(t_{m} ; R_{B}\right)=\sqrt{R_{0}^{2}+\left[V\left(t_{m}-t_{c}\right)\right]^{2}+2 R_{0} V\left(t_{m}-t_{c}\right) \sin \theta_{s q}}, \\
R_{0}=\frac{R_{B}}{\cos \theta_{s q}} .
\end{gathered}
$$

\subsection{The SAS echo signal of target point}

Assume that the following linear frequency modulated signal is transmitted by SAS:

$$
s_{t}=w(\hat{t}) \exp \left\{j \pi K_{r} \hat{t}^{2}\right\},
$$

where $w(\hat{t})$ is the signal envelope, $K_{r}$ is the FM range, and $\hat{t}$ is the time.

The model of the target baseband signal in the range between the fast-time and azimuth slow-time domain $\left(\hat{t}-t_{m}\right.$ domain) is: 


$$
\begin{gathered}
s_{0}\left(\hat{t}, t_{m} ; R_{B}\right)=A_{0} w_{r}\left[\hat{t}-2 R\left(t_{m} ; R_{B}\right) / c\right] w_{a}\left(t_{m}-t_{c}\right) \times \\
\times \exp \left\{-j \pi \frac{4 R\left(t_{m} ; R_{B}\right)}{\lambda}\right\} \times \exp \left\{j \pi K_{r}\left[\hat{t}-\frac{2 R\left(t_{m} ; R_{B}\right)}{c}\right]^{2}\right\},
\end{gathered}
$$

where $A_{0}$ is an arbitrary complex constant, $w_{r}(t)$ is the range envelope (the rectangular window function), $w_{a}(t)$ is the azimuth envelope (the $\sin c$ quadric function), and $\lambda$ is the signal wavelength.

\subsection{D spectrum of the target point SAS echo signal}

By double application of the principle of the stationary phase (POSP) to the Equation (4), the signal can be transformed from the 2D time domain into the $2 \mathrm{D}$ frequency domain:

$$
\mathrm{SS}\left(f_{r}, f_{a} ; R_{B}\right)=A_{0} \exp \left\{j \phi\left(f_{r}, f_{a} ; R_{B}\right)-j \frac{\pi}{K_{r}} f_{r}^{2}\right\},
$$

where $f_{r}$ and $f_{a}$ represent the range frequency and azimuth frequency, respectively. The value of $f_{r}$ cannot exceed the bandwidth of the transmitted signal $B_{r}$, and the value of $f_{a}$ cannot exceed the Doppler bandwidth $B_{a}$, i.e. $\left|f_{r}\right| \leq B_{r}$ and $\left|f_{a}\right| \leq B_{a}$. The phase factor $\varphi\left(f_{r}, f_{a} ; R_{B}\right)$ is defined by:

$$
\begin{gathered}
\phi\left(f_{r}, f_{a} ; R_{B}\right)=-\frac{4 \pi R_{B}}{\lambda} \sqrt{\left(1+\frac{f_{r}}{f_{c}}\right)^{2}-\left(\frac{\lambda f_{a}}{2 V}\right)^{2}}= \\
=-\frac{4 \pi R_{B}}{\lambda} \sqrt{1-\left(\frac{\lambda f_{a}}{2 V}\right)^{2}}\left[1+\frac{\frac{2 f_{r}}{f_{c}}+\left(\frac{f_{r}}{f_{c}}\right)^{2}}{1-\left(\frac{\lambda f_{a}}{2 V}\right)^{2}}\right]^{\frac{1}{2}},
\end{gathered}
$$

where $f_{c}$ is the sonar operating frequency. In the derivation above, the squint angle approximation is used. Therefore, as long as the trajectory is represented in form of the hyperbolic curve, the phase factor is the accurate expression of the signal spectrum phase at any squint angle.

\subsection{Phase compensation of the SAS echo}

The basic idea of the SAS imaging algorithms is to divide 2D echo spectrum into 1D spectrums in range direction and azimuth direction, and process them separately, which generally represents the process of constructing the frequency or time domain phase compensation factor using the frequency spectra characteristic of the echo. In the case of the side-looking or small squint angle phase compensation factors' accuracy requirements are low, while in the case of the large squint angle the requirements are very high due to the severe range-azimuth coupling. 
If Equation (6) is expanded to its Taylor series at $\frac{f_{r}}{f_{c}}$ it can be rewritten as

$$
\begin{gathered}
\phi\left(f_{r}, f_{a} ; R_{B}\right)=-\frac{4 \pi R_{B}}{\lambda} \sqrt{1-\left(\frac{\lambda f_{a}}{2 V}\right)^{2}}\left[1+\frac{\frac{2 f_{r}}{f_{c}}+\left(\frac{f_{r}}{f_{c}}\right)^{2}}{1-\left(\frac{\lambda f_{a}}{2 V}\right)^{2}}\right]^{\frac{1}{2}}= \\
=-\frac{4 \pi R_{B}}{\lambda} D\left(f_{a}, V\right)-\frac{2 \pi R_{0}}{\lambda} \frac{1}{D\left(f_{a}, V\right)} \frac{f_{r}}{f_{c}}\left(2+\frac{f_{r}}{f_{c}}\right)^{+} \\
+\frac{\pi R_{0}}{2 \lambda} \frac{1}{D\left(f_{a}, V\right)}\left[\frac{f_{r}}{f_{c}}\left(2+\frac{f_{r}}{f_{c}}\right)\right]^{2}+\cdots
\end{gathered}
$$

Furthermore, if the Equation (7) is reorganized and all terms whose order is higher than second order omitted, we get

$$
\text { (8) } \begin{gathered}
\phi\left(f_{r}, f_{a} ; R_{B}\right)=p_{0}\left(f_{a}, R_{B}\right)+p_{1}\left(f_{a}, R_{B}\right) \frac{f_{r}}{f_{c}}+p_{2}\left(f_{a}, R_{B}\right)\left(\frac{f_{r}}{f_{c}}\right)^{2}+p_{3}\left(f_{a}, R_{B}\right)\left(\frac{f_{r}}{f_{c}}\right)^{3}= \\
=-\frac{4 \pi R_{B}}{\lambda} D\left(f_{a}, V\right)-\frac{4 \pi R_{B}}{\lambda} \frac{1}{D\left(f_{a}, V\right)} \frac{f_{r}}{f_{c}}+ \\
+\frac{2 \pi R_{B}}{\lambda} \frac{1-D^{2}\left(f_{a}, V\right)}{D^{3}\left(f_{a}, V\right)}\left(\frac{f_{r}}{f_{c}}\right)^{2}-\frac{\pi R_{B}}{2 \lambda} \frac{4-D^{2}\left(f_{a}, V\right)}{D^{5}\left(f_{a}, V\right)}\left(\frac{f_{r}}{f_{c}}\right)^{3},
\end{gathered}
$$

where $p_{0}\left(f_{a}, R_{B}\right), p_{1}\left(f_{a}, R_{B}\right), p_{2}\left(f_{a}, R_{B}\right)$ and $p_{3}\left(f_{a}, R_{B}\right)$ are the zero-order, the firstorder, the second-order and the third-order coefficients of $\frac{f_{r}}{f_{c}}$, respectively, and $D\left(f_{a}, V\right)=\sqrt{1-\frac{c^{2} f_{a}^{2}}{4 \mathrm{~V}^{2} f_{c}^{2}}}=\sqrt{1-\left(\frac{\lambda f_{a}}{2 V}\right)^{2}}$ presents the migration factor.

\section{Improved CS Algorithm}

CS Algorithm can only handle small squint angle imaging so it is limited to narrowband and narrow-beam signals. The wide-band and wide-beam SAS signals can be also processed by the CS Algorithm but with less effectiveness. The Improved CS (ICS) Algorithm proposed in this paper is suitable also for wide-band and wide-beam SAS signals, and even in the case of the large squint angle it can compensate for higher-order phase of the echo, thus achieving accurate target focusing.

The algorithm is mainly based on the phase multiplication and the Fast Fourier Transform (FFT). ICS Algorithm steps are the following: 
Step 1. Azimuth and range FFT

The echo is transformed to 2D frequency domain signal by 2D FFT as presented in Equation (5).

Step 2. 2D frequency domain phase compensation for reference point and thirdorder phase compensation of the imaging area

In 2D frequency domain, phase compensation for the reference point and thirdorder phase compensation for the imaging area are implemented. When the standard CS Algorithm is used for the wide-band high-resolution SAS signal, the Taylor series expansion gives errors and results in reduced effective sonar imaging range with the decreasing of carrier frequency. The main reason for that phenomenon is that the target point in azimuth direction is no longer a linear FM signal. If we assume that $Y\left(f_{a}\right)$ is the third-order phase filtering coefficient, the cubic filtering function could be constructed as

$$
H_{\text {third }}\left(f_{r}, f_{a}\right)=\exp \left\{j \frac{2 \pi}{3} Y\left(f_{a}\right) f_{r}^{3}\right\} .
$$

If the complex constants irrelevant to the derivation are ignored, and Equation (5) is multiplied by Equation (9), we get:

$$
\begin{gathered}
\mathrm{SS}_{\mathrm{ICS}}\left(f_{r}, f_{a} ; R_{B}\right)= \\
=\exp \left\{j \varphi_{\text {third }}\left(f_{r}, f_{a} ; R_{B}\right)\right\} \exp \left\{-j \frac{\pi}{K} f_{r}^{2}\right\} \exp \left\{j \frac{2 \pi}{3} Y\left(f_{a}\right) f_{r}^{3}\right\},
\end{gathered}
$$

where the coefficient of $f_{r}^{3}$ can be expressed as $Y_{m}\left(f_{a}\right)=Y\left(f_{a}\right)+\frac{3 p_{3}\left(f_{a}, R_{\mathrm{ref}}\right)}{2 \pi f_{c}^{3}}$.

Step 3. Range Inverse FFT (IFFT)

Application of the POSP and complex constants ignoring lead to the following expression for the echo in Doppler domain:

$$
\begin{gathered}
s S_{\mathrm{ICS}}\left(f_{r}, f_{a} ; R_{B}\right)=\exp \left[-j \pi \frac{4 R_{B}}{\lambda} D\left(f_{a}, V\right)\right] \times \\
\times \exp \left\{j \pi K_{s}\left(f_{a}, R_{\mathrm{ref}}\right)\left[\hat{t}-\frac{2}{c} R_{B}\left[1+C_{s}\left(f_{a}\right)\right]\right]^{2}\right\} \times \\
\times \exp \left\{j \pi \frac{2 \pi}{3} Y_{m}\left(f_{a}\right) K_{s}^{2}\left(f_{a}, R_{\mathrm{ref}}\right)\left[\widehat{t}-\frac{2}{c} R_{B}\left[1+C_{s}\left(f_{a}\right)\right]\right]^{2}\right\} .
\end{gathered}
$$

Step 4. Multiplication of the CS factor in order to eliminate range cell migration The multiplication of the CS factor by Equation (11) gives

$$
H_{\mathrm{ICS}}\left(\widehat{t}, f_{a} ; R_{\mathrm{ref}}\right)=
$$

$$
=\exp \left\{j \pi q_{2}\left(f_{a}\right)\left[\hat{t}-\frac{2}{c} R_{\text {ref }}\left[1+C_{s}\left(f_{a}\right)\right]\right]^{2}\right\} \exp \left\{j \pi q_{3}\left(f_{a}\right)\left[\hat{t}-\frac{2}{c} R_{\text {ref }}\left[1+C_{s}\left(f_{a}\right)\right]^{3}\right\},\right.
$$

where $q_{2}\left(f_{a}\right)$ and $q_{3}\left(f_{a}\right)$ are the coefficients which should to be determined. 
Step 5. Range compression and RCMC

After the CS signal processing the POSP is applied to the echo signal in order to convert it to 2D frequency domain and to expand the phase functions into the power series of range frequency $f_{r}$. The first-order term of $f_{r}$ corresponds to the range position and to the RCM of the target point. The representation of the first-order term coefficient into series of $\left(R_{B}+R_{\mathrm{ref}} C_{s}\left(f_{a}\right)\right)$, and setting of the second-order term coefficient to zero, will make the RCM curve not space-variant. The quadratic term of $f_{r}$ corresponds to the range frequency. Moreover, the representation of the quadratic term coefficient as series of $\left(R_{B}+R_{\text {ref }} C_{s}\left(f_{a}\right)\right)$ and setting of the first-order term to zero will eliminate the secondary range compression dependence on the range. Then, the undetermined coefficients mentioned above can be obtained as follows:

$$
\begin{gathered}
q_{2}\left(f_{a}\right)=K_{s}\left(f_{a}, R_{\mathrm{ref}}\right) C_{s}\left(f_{a}\right), \\
q_{3}\left(f_{a}\right)=K_{s}^{2}\left(f_{a}, R_{\mathrm{ref}}\right)\left[1-D\left(f_{a}, V\right)\right] \frac{\lambda}{3 c} \frac{1-D^{2}\left(f_{a}, V\right)}{D^{3}\left(f_{a}, V\right)}, \\
Y\left(f_{a}\right)=\frac{\lambda}{2 c K_{s}\left(f_{a}, R_{\mathrm{ref}}\right)} \frac{\left[1+D\left(f_{a}, V\right)\right]\left[2-D\left(f_{a}, V\right)\right]}{D^{2}\left(f_{a}, V\right)}-\frac{3 p_{3}\left(f_{a}, R_{\mathrm{ref}}\right)}{2 \pi f_{c}^{3}} .
\end{gathered}
$$

After the CS processing 2-D spectrum of the target echo becomes:

$$
\begin{gathered}
\mathrm{SS}_{\mathrm{ICS}}\left(f_{r}, f_{a} ; R_{B}\right)=\exp \left\{-j \pi \frac{4}{c}\left[R_{B}+R_{\mathrm{ref}} C_{s}\left(f_{a}\right) f_{r}\right]\right\} \times \\
\times \exp \left\{-j \pi \frac{D\left(f_{a}, V\right)}{K_{s}\left(f_{a}, R_{\mathrm{ref}}\right)} f_{r}^{2}\right\} \times \exp \left\{-j \pi \frac{4 R_{B}}{\lambda} D\left(f_{a}\right)\right\} \times \exp j \phi^{\prime}\left(f_{a}, R_{B}\right) .
\end{gathered}
$$

Moreover, in derivation of Equation (14) the complex constants are ignored, and $\phi^{\prime}\left(f_{a}, R_{B}\right)$ is

$$
\phi^{\prime}\left(f_{a}, R_{B}\right)=\psi_{1}\left(f_{a}, R_{B}\right)+\psi_{2}\left(f_{a}, R_{B}\right)+\psi_{3}\left(f_{a}, f_{r}\right),
$$

where $\psi_{1}\left(f_{a}, R_{B}\right), \psi_{2}\left(f_{a}, R_{B}\right)$ and $\psi_{3}\left(f_{a}, R_{B}\right)$ are:

$$
\begin{gathered}
\psi_{1}\left(f_{a}, R_{B}\right)=\frac{4 \pi}{c^{2}} K_{s}\left(f_{a}, R_{r e f}\right)\left[1+D\left(f_{a}, V\right)\right] D\left(f_{a}, V\right) R_{B}-R_{\text {ref }}{ }^{2}, \\
\psi_{2}\left(f_{a}, R_{B}\right)=\frac{8 \pi \lambda}{c^{4}} K_{s}^{2}\left(f_{a}, R_{\mathrm{ref}}\right) \frac{\left[1-D\left(f_{a}, V\right)\right]\left[1-D^{2}\left(f_{a}, V\right)\right]}{D^{5}\left(f_{a}, V\right)} R_{B}-R_{\mathrm{ref}}{ }^{3}, \\
\psi_{3}\left(f_{a}, R_{B}\right)=\frac{\pi \lambda}{3 c K_{s}\left(f_{a}, R_{\mathrm{ref}}\right)}\left[1-D\left(f_{a}, V\right)\right]\left[1-D^{2}\left(f_{a}, V\right)\right]+ \\
+\left[1+D\left(f_{a}, V\right)\right]\left[2-D\left(f_{a}, V\right)\right] D\left(f_{a}, V\right) f_{r}^{3}
\end{gathered}
$$

where $\psi_{1}\left(f_{a}, R_{B}\right)$ is the range compression residual phase; $\psi_{2}\left(f_{a}, R_{B}\right)$ is the range compression residual phase of the third-order phase filtering, and $\psi_{3}\left(f_{a}, R_{B}\right)$ is also 
the product of third-order phase filtering, used to correct range compression factor. By compensating of the terms of $f_{r}$ and the $\psi_{3}\left(f_{a}, R_{B}\right)$ terms in (16), the range compression, the second range compression and the RCMC are completed, where the compensation factor is

$$
H_{c 1}\left(f_{r}, f_{a} ; R_{\mathrm{ref}}\right)=\exp \left\{j \pi \frac{4 R_{\mathrm{ref}} C_{s}\left(f_{a}\right) f_{r}}{c}\right\} \exp \left\{j \pi \frac{D\left(f_{a}, V\right)}{K_{s}\left(f_{a}, R_{\mathrm{ref}}\right)} f_{r}^{2}\right\} \exp \left\{-j \phi_{3}\left(f_{a}, R_{B}\right)\right\} .
$$

Step 6. Azimuth focusing and residual phase error compensation

The previous result is transformed to Doppler domain through the range IFFT in order to accomplish the azimuth focusing and phase error compensation, i.e., to compensate $\psi_{1}\left(f_{a}, R_{B}\right), \psi_{2}\left(f_{a}, R_{B}\right)$ and azimuth compression factor. The factor is

$$
H_{c 2}\left(f_{r}, f_{a} ; R_{\mathrm{ref}}\right)=\exp \left\{-j\left[\phi_{1}\left(f_{a}, R_{B}\right)+\phi_{2}\left(f_{a}, R_{B}\right)-\frac{4 \pi R_{B}}{\lambda}\right]\right\} \text {. }
$$

Step 7. Azimuth IFFT

After azimuth IFFT, the final image is obtained as

$$
\operatorname{ss}\left(\hat{t}, t_{m} ; R_{B}\right)=C \sin c\left\{\Delta f_{r}\left(\hat{t}-\frac{2 R_{B}}{c}\right)\right\} \sin c\left(\Delta f_{a} t_{m}\right),
$$

where $\Delta f_{a}$ is the Doppler bandwidth by $\Delta f_{a}=K_{r} T_{s}$, and $T_{s}$ is the synthetic aperture time.

\section{Algorithm simulation}

The target consists of three scattering points whose coordinates are the following: $(0,38),(5,45)$, and $(-2,42)$, where the horizontal coordinate refers to the azimuth, and the vertical coordinate refers to the range. The imaging of all three scattering points is carried out both in side-looking mode and squint mode using the Conventional CS (CCS) Algorithm, and after that the imaging at the same conditions is also conducted using ICS Algorithm.

Table 1. Point targets simulation parameter
\begin{tabular}{|l|c|}
\hline \multicolumn{1}{|c|}{ Parameter } & Value \\
\hline Center frequency (kHz) & 50 \\
\hline Platform velocity (m/s) & 1 \\
\hline Pulse width (ms) & 120 \\
\hline Pulse repetition frequency (Hz) & 10 \\
\hline Signal bandwidth (kHz) & 20 \\
\hline Array element width (m) & 0.2 \\
\hline Squint angle ${ }^{\circ}{ }^{\circ}$ & $5,10,15$ \\
\hline
\end{tabular}

Fig. 2 is a side-looking mode, using the Classical CS Algorithm of three point target imaging effect. As can be seen in the side-looking mode, the CCS Algorithm 
can acquire high quality imaging effect. Fig. 4 has showed the CCS Algorithm imaging effect when the squint angel is $5^{\circ}$. Compared with the Figs 2 and 3, it is known that range-azimuth coupling leads the image defocusing because of the squint mode. Figs 4 and 5 is for CCS Algorithm squint mode imaging effect when the squint angle is $10^{\circ}, 15^{\circ}$. It can be seen from the imaging results, the range-azimuth coupling degree is more and more serious with the increase of the squint angle. The CCS Algorithm cannot completely eliminate the coupling, which leads to more obvious image defocusing. Figs 6-8 are respectively shown the ICS Algorithm imaging effect when the squint angle is $5^{\circ}, 10^{\circ}$ and $15^{\circ}$. Compared with the CCS Algorithm, the ICS Algorithm can eliminate the range-azimuth coupling more completely, so it can acquire more quality imaging.

Table 2. Performance comparison of CCS and ICS Algorithm

\begin{tabular}{|c|c|c|c|c|}
\hline \multirow{3}{*}{$\begin{array}{l}\text { Squint } \\
\text { angle }\end{array}$} & \multicolumn{2}{|c|}{ CCS Algorithm } & \multicolumn{2}{c|}{ ICS Algorithm } \\
\cline { 2 - 5 } & Azimuth (dB) & Range (dB) & Azimuth (dB) & Range (dB) \\
\cline { 2 - 5 } & PSLR & ISLR & PSLR & ISLR \\
\hline $0^{\circ}$ & -12.153 & -9.565 & -12.012 & -9.503 \\
\hline $5^{\circ}$ & -12.395 & -9.623 & -12.113 & -9.553 \\
\hline $10^{\circ}$ & -13.595 & -10.728 & -12.236 & -9.869 \\
\hline $15^{\circ}$ & -14.676 & -11.435 & -12.382 & -9.902 \\
\hline
\end{tabular}

The $3 \mathrm{~dB}$ main slob width, the Integral Side Lobe Ratio (ISLR), and the Peak Side Lobe Ratio (PSLR) of the point target are compared assuming that the matching functions are not window functions. The general imaging requirements are ISLR $<-10 \mathrm{~dB}$, and PSLR $<-15 \mathrm{~dB}$. Table 1 shows range and azimuth ISLR and PSLR for all three target points for different squint angles. Table 2 compares the performance of the CCS Algorithm and the ICS Algorithm at target point $(5 \mathrm{~m}, 45 \mathrm{~m})$ in terms of the PSLR and the ISLR. From the results presented above, we can conclude that the new processing method provides image quality better than CCS Algorithm.

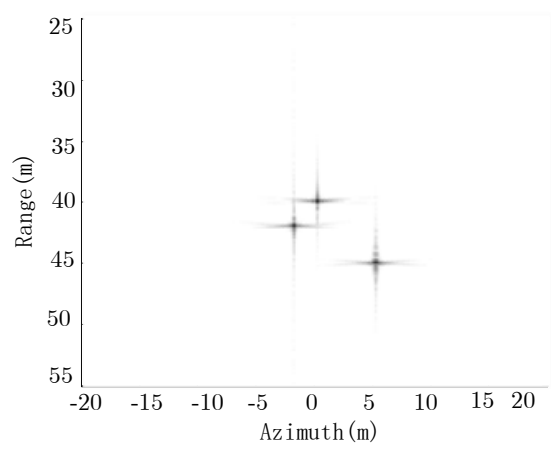

Fig. 2. Side-looking mode imaging 


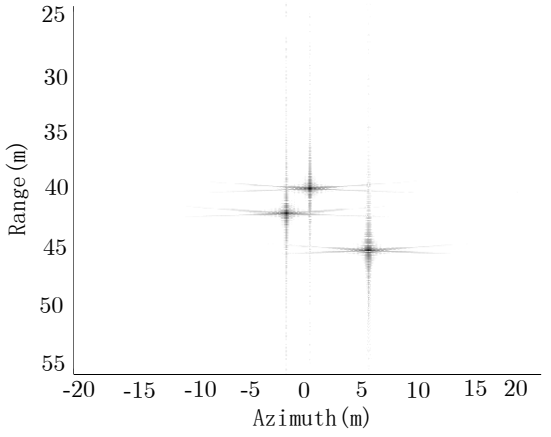

Fig. 3. CCS Algorithm (squint angle $=5^{\circ}$ )

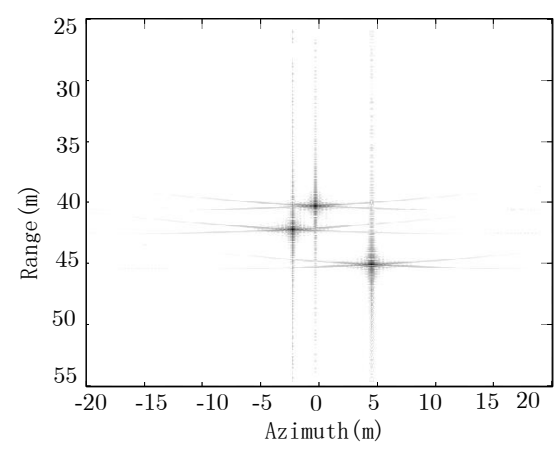

Fig. 4. CCS Algorithm (squint angle $=10^{\circ}$ )

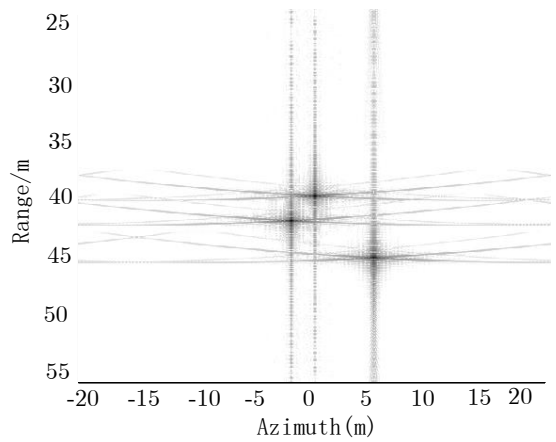

Fig. 5. CCS Algorithm (squint angle $=15^{\circ}$ ) 


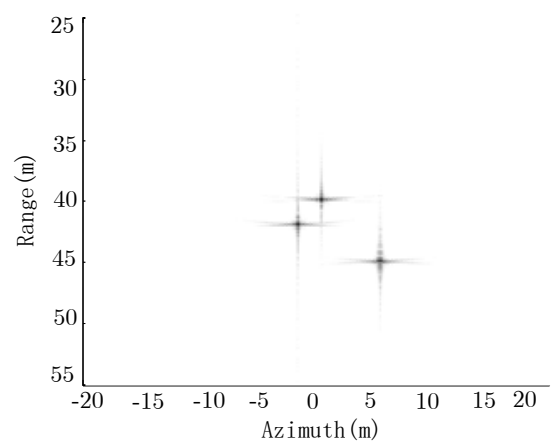

Fig. 6. ICS Algorithm (squint angle $=5^{\circ}$ )

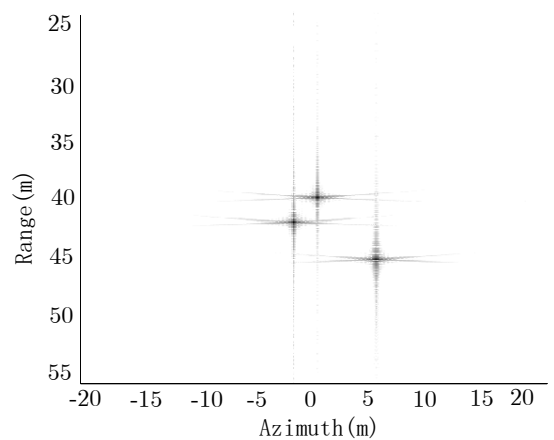

Fig. 7. ICS Algorithm (squint angle $=10^{\circ}$ )

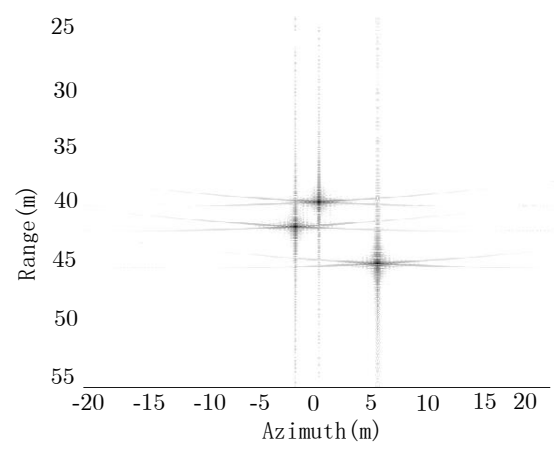

Fig. 8. ICS Algorithm (squint angle $=15^{\circ}$ )

\section{Conclusion}

In the case of the large squint angle, the SAS imaging suffers from severe rangeazimuth coupling, which makes the RCMC more difficult and has big influence on the image focusing. This paper presents analysis of the range-azimuth coupling 
relationship in 2D frequency domain; the more accurate azimuth modulation term and coupling terms of different orders are obtained. Through the CS scaling of the RCMC and the higher-order echo phase compensation, the migration trajectories of all the targets in the band are accurately corrected. Finally, azimuth focusing is realized through the improved matched filtering function. Simulation results have shown that the improved algorithm significantly enhanced the SAS imaging performances in the squint mode and that the proposed algorithm can meet the requirements of the high-resolution imaging in the case of large squint angle.

\section{References}

1. C a 11 o w, H. J. Signal Processing for Synthetic Aperture Sonar Image Enhancement. - University of Canterbury, Christchurch, New Zealand, 2003, Vol. 4, pp. 32-34.

2. N a m l e r, R., H. R u n g e. A Novel Processing SAR Focusing Algorithm Based on Chirp Scaling. - In: Proc. of IGARSS'92, Clear Lake, TX, May 1992, pp. 372-375.

3. P e n g, S.-Y. The Key Technology Research on Missile-Bornesynthetic Aperture Radar Imaging. National University of Defense Technology, 2011.

4. W u, Y., H.-J. S o n g, J. P e n g. Chirp Scaling Imaging Algorithm of SAR in High Squint Mode Based on Range Walk Removal. - Jounal of Electronics \& Information Technology, Vol. 32, 2010, No 3, pp. 593-598.

5. X i o n g, J., et al. Simulation Study of Imaging for Squint-Looking Airborne SAR Based on RWC. - Fire Control \& Command Control, Vol. 37, 2012, No 4, pp. 176-180.

6. Z h a n g, S.-X., et al. Focus Improvement of High-Squint SAR Based on Azimuth Dependence of Quadratic Range Cell Migration Correction. - IEEE Geoscience and Remote Sensing Letters, Vol. 10, 2013, No 1, pp. 150-154.

7. A n, D.-X., et al. Extended Nonlinear Chirp Scaling Algorithm for High-Resolution Highly Squint SAR Data Focusing. - IEEE Transactions on Geoscience and Remote Sensing, Vol. 50, 2012, No 9, pp. 3595-3609.

8. L i u, G.-G. Focusing Highly Squinted Data Using the Extended Nonlinear Chirp Scaling Algorithm. - IEEE Geoscience and Remote Sensing Letters, Vol. 10, 2013, No 2, pp. 342-346.

9. Z h a n g, X., et al. Chirp-Scaling Imaging Algorithm for Multi-Receiver Synthetic Aperture Sonar. - Systems Engineering and Electronics, 2013, No 7, pp. 1415-1420.

10. L i u, W., et al. A Multiple-Receiver Synthetic Aperture Sonar CS Imaging Algorithm. - Technical Acoustics, 2008, No 10, pp. 636-640.

11. Z h a n g, X., et al. Multireceiver Correction for the Chirp Scaling Algorithm in Synthetic Aperture Sonar. - IEEE Journal of Oceanic Engineering, 2014, No 7, pp. 472-481.

12. F a n, N., et al. Research on Modified Range-Doppler Algorithm of Synthetic Aperture Sonar Imaging. - Computer Simulation, 2014, No 10, pp. 25-29. 